\title{
Versione italiana dello Starvation Symptom Inventory: un questionario per valutare i sintomi da malnutrizione nei disturbi dell'alimentazione
}

\author{
Simona Calugi', Selvaggia Sermattei ${ }^{2}$, Riccardo Dalle Grave ${ }^{1}$ \\ ${ }^{1}$ Department of Eating and Weight Disorders, Villa Garda Hospital \\ ${ }^{2}$ Anoressia, Bulimia e Obesità, Firenze
}

\section{Parole chiave}

Anoressia nervosa,

Disturbi dell'alimentazione

Sintomi da malnutrizione

Restrizione dietetica

Validità

Affidabilità

Analisi fattoriale

\begin{abstract}
Riassunto
Introduzione. I sintomi da malnutrizione presenti nelle persone con anoressia nervosa determinano profonde modificazioni della personalità e contribuiscono a mantenere la psicopatologia del disturbo dell'alimentazione. Scopo di questo studio è descrivere il processo di costruzione e validazione della versione italiana dello Starvation Symptom Inventory (SSI).
\end{abstract}

Metodi. Sono stati reclutati 150 pazienti di sesso femminile con anoressia nervosa, 30 pazienti con disturbo dell'alimentazione non sottopeso, 15 pazienti con disturbo bipolare in fase depressiva e 341 controlli sani. I pazienti con anoressia nervosa hanno completato l'Eating Disorder Examination Questionnaire e il Brief Symptoms Inventory, mentre tutti i partecipanti hanno valutato i loro sintomi da malnutrizione in una scala Likert (0-6) riportando il numero di giorni in cui hanno sperimentato tali sintomi negli ultimi 28 giorni.

Risultati. L'analisi fattoriale dello SSI sul campione di pazienti con anoressia nervosa ha individuato un singolo fattore che spiega circa la metà della varianza. II questionario ha dimostrato una buona consistenza interna $(\alpha=0,91)$ e affidabilità test-retest $(r=0,90)$. II punteggio globale dello SSI è risultato significativamente correlato con la psicopatologia dei disturbi dell'alimentazione e la psicopatologia generale, dimostrando una buona validità convergente. I punteggi dello SSI sono risultati significativamente più elevati nei pazienti affetti da anoressia nervosa rispetto ai controlli sani, ai pazienti non sottopeso con disturbi dell'alimentazione e ai pazienti con disturbo bipolare in fase depressiva.

Conclusioni. La versione italiana dello SSI è promettente per valutare i sintomi da malnutrizione e le loro modificazioni durante il recupero ponderale nei pazienti con anoressia nervosa e può essere facilmente integrata nella pratica clinica.

\begin{abstract}
Introduction. Starvation symptoms in people with anorexia nervosa determine profound personality changes and contribute to maintaining the psychopathology of eating disorders. The aim of this study is to describe the design and validation of the Italian version of the Starvation Symptom Inventory (SSI).

Methods. 150 female patients with anorexia nervosa, 30 patients with non-underweight eating disorder, 15 patients with bipolar disorder in depressive phase and 341 healthy controls were recruited. Patients with anorexia nervosa completed the Eating Disorder Examination Questionnaire and the Brief Symptoms Inventory, while all participants evaluated their starvation symptoms on a Likert scale (0-6) reporting the number of days in which they had experienced them in the previous 28 days.

Results. The factorial analysis of the SSI on the sample of patients with anorexia nervosa identified a single factor that explains about half of the variance. The questionnaire showed a good internal consistency $(\alpha=0.91)$ and reliability test-retest $(r=0.90)$. The overall SSI score was significantly correlated with the psychopathology of eating disorders and the general psychopathology, demonstrating a good convergent validity. SSI scores were significantly higher in patients with anorexia nervosa than healthy controls, non-underweight patients with eating disorder and patients with bipolar disorder in the depressive phase.
\end{abstract}

Conclusions. The Italian version of the SSI is a promising tool for assessing symptoms of starvation and their change during weight restoration in patients with anorexia nervosa that can be easily integrated into clinical practice.

Copyright () 2019 Simona Calugi et al. This is an open-access article distributed under the terms of the Creative Commons Attribution License (CC BY). The use, distribution or reproduction in other forums is permitted, provided the original author(s) and the copyright owner(s) are credited and that the original publication in this journal is cited, in accordance with accepted academic practice. No use, distribution or reproduction is permitted which does not comply with these terms.

Simona Calugi, PhD (凶) si.calugi@gmail.com

Ricevuto: 15 Gennaio 2019; Accettato: 1 Febbraio 2019; Pubblicato online: 15 Febbraio 2019. doi:10.32044/ijedo.2019.02 


\section{Introduzione}

Il Minnesota Starvation Experiment è il più importante studio che ha valutato gli effetti della restrizione alimentare calorica e della perdita di peso nelle persone normopeso. Lo studio è stato condotto da Ancel Keys e collaboratori nell'Università del Minnesota (Keys, Brozek, Henschel, Mickelsen, \& Taylor, 1950). Più di 100 uomini si offrirono volontari per lo studio come alternativa al servizio militare; i 36 uomini selezionati possedevano sia le migliori condizioni di salute fisica e psicologica, sia il maggiore impegno verso gli obiettivi dell'esperimento. Durante i primi tre mesi dell'esperimento, i volontari si cibarono normalmente mentre venivano studiati nel dettaglio il loro comportamento, la loro personalità e le loro modalità alimentari. Nel corso dei sei mesi successivi, i partecipanti furono sottoposti a una restrizione che corrispondeva approssimativamente alla metà del loro introito calorico iniziale; questo regime determinò una perdita approssimativa del $25 \%$ del loro peso iniziale. I sei mesi di perdita di peso furono seguiti da tre mesi di riabilitazione nutrizionale, durante i quali gli uomini furono gradualmente nutriti in maniera normale. Un sottogruppo fu seguito per quasi nove mesi dopo che era iniziata la fase di nutrizione normale.

La maggior parte dei risultati fu riportata solo per 32 uomini, dato che quattro dei partecipanti all'esperimento si erano ritirati o durante o alla fine della fase di restrizione calorica. Nonostante le risposte individuali, rispetto alla perdita di peso, variassero notevolmente, tutti gli uomini sperimentarono rilevanti cambiamenti fisici, psicologici e sociali (Keys et al., 1950). I principali sintomi da malnutrizione (per difetto) riportati dai volontari riguardavano attitudini e comportamenti anomali nei confronti del cibo e dell'alimentazione (per es. preoccupazione per l'alimentazione, caratteristici rituali alimentari, collezioni di ricette e libri di cucina, incremento del consumo di caffè, tè, spezie, gomme da masticare e acqua, episodi di abbuffata), alterazioni emotive e sociali (per es. danneggiamento della concentrazione, depressione, sbalzi del tono dell'umore, irritabilità, rabbia, ansia, apatia, indecisione, rigidità, isolamento sociale, diminuzione del desiderio sessuale) e sintomi fisici (per es. sensazione di pienezza precoce, dispepsia, intolleranza al freddo, disturbi del sonno). Lo studio ha avuto ampio risalto nella stampa e il 30 luglio 1945 l'edizione della rivista Life ha pubblicato un articolo dal titolo "Men Starve in Minnesota", con diverse fotografie impressionanti dei volontari (Figura 1) (Kalm \& Semba, 2005).

Losservazione clinica che molti dei sintomi riportati dai volontari del Minnesota Starvation Experiment sono simili a quelli riferiti dai pazienti con anoressia nervosa, ha migliorato la comprensione e la gestione dei disturbi dell'alimentazione e oggi è ampiamente accettato che molti sintomi, una volta attribuiti alla psicopatologia specifica dell'anoressia nervosa, sono la mera conseguenza della malnutrizione e si risolvono con la riabilitazione nutrizionale (Dalle Grave, Pasqualoni, \& Marchesini, 2011; Garner, 1977).

Più recentemente, la teoria cognitivo comportamentale transdiagnostica ha sostenuto che i sintomi da malnutrizione giocano un ruolo centrale nel mantenere la psicopatologia dei disturbi dell'alimentazione (Fairburn, 2008).

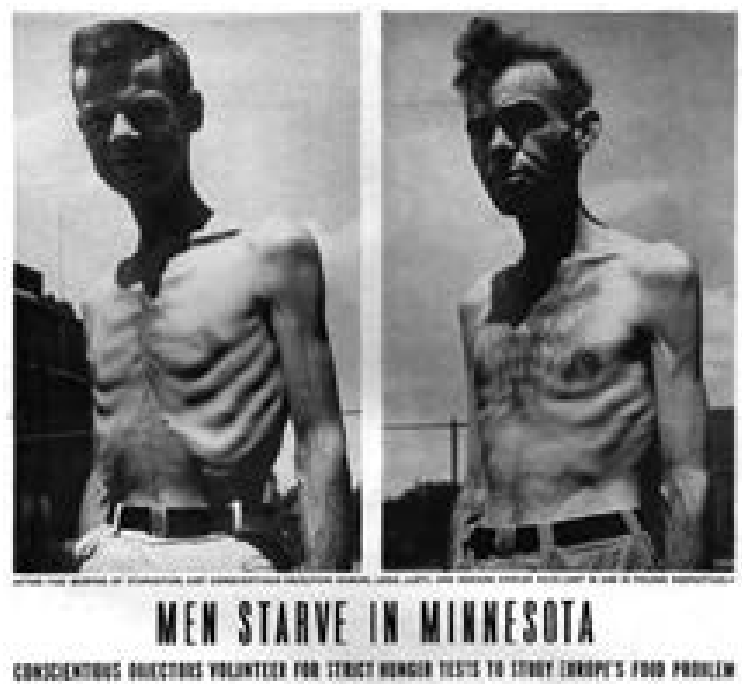

Figura 1. Fotografia della rivista Life che riprende gli obiettori di coscienza durante il Minnesota Starvation Experiment. July 30, 1945. Volume 19, Number 5, p. 43. Credit: Wallace Kirkland/Time Life Pictures/Getty Images 
Infatti, se i sintomi da malnutrizione negli individui senza disturbi dell'alimentazione hanno la funzione positiva di focalizzare l'attenzione primariamente sulla ricerca del cibo e di prolungare l'esistenza attraverso una riduzione del metabolismo basale, questo non accade nelle persone affette da disturbi dell'alimentazione (Dalle Grave, Calugi, \& Sartirana, 2018). Due studi recenti hanno infatti evidenziato che gli effetti del sottopeso e della restrizione calorica non si limitano solo a causare dei sintomi fisici e psicosociali che ostacolano il raggiungimento di un normale funzionamento psicologico, ma svolgono un ruolo importante nel mantenere il disturbo dell'alimentazione (Dalle Grave, Di Pauli, Sartirana, Calugi, \& Shafran, 2007; Shafran, Fairburn, Nelson, \& Robinson, 2003). Per esempio, alcuni sintomi da malnutrizione interagendo con la psicopatologia del disturbo dell'alimentazione, sono interpretati come minaccia al controllo alimentare (per es. la fame) o come fallimento del controllo alimentare (per es. il precoce senso di pienezza), mentre altri, come la diminuzione della socializzazione, isolano l'individuo da esperienze esterne che potrebbero ridurre l'eccessiva valutazione del peso, della forma del corpo e del controllo dell'alimentazione. Alcuni pazienti, infine, interpretano alcuni sintomi da malnutrizione positivamente (per es., la fame, le vertigini, la debolezza e il sentire freddo) come il segno che stanno avendo successo nel controllare l'alimentazione. La Figura 2 illustra alcuni meccanismi con cui i sintomi da malnutrizione mantengono la psicopatologia del disturbo dell'alimentazione.

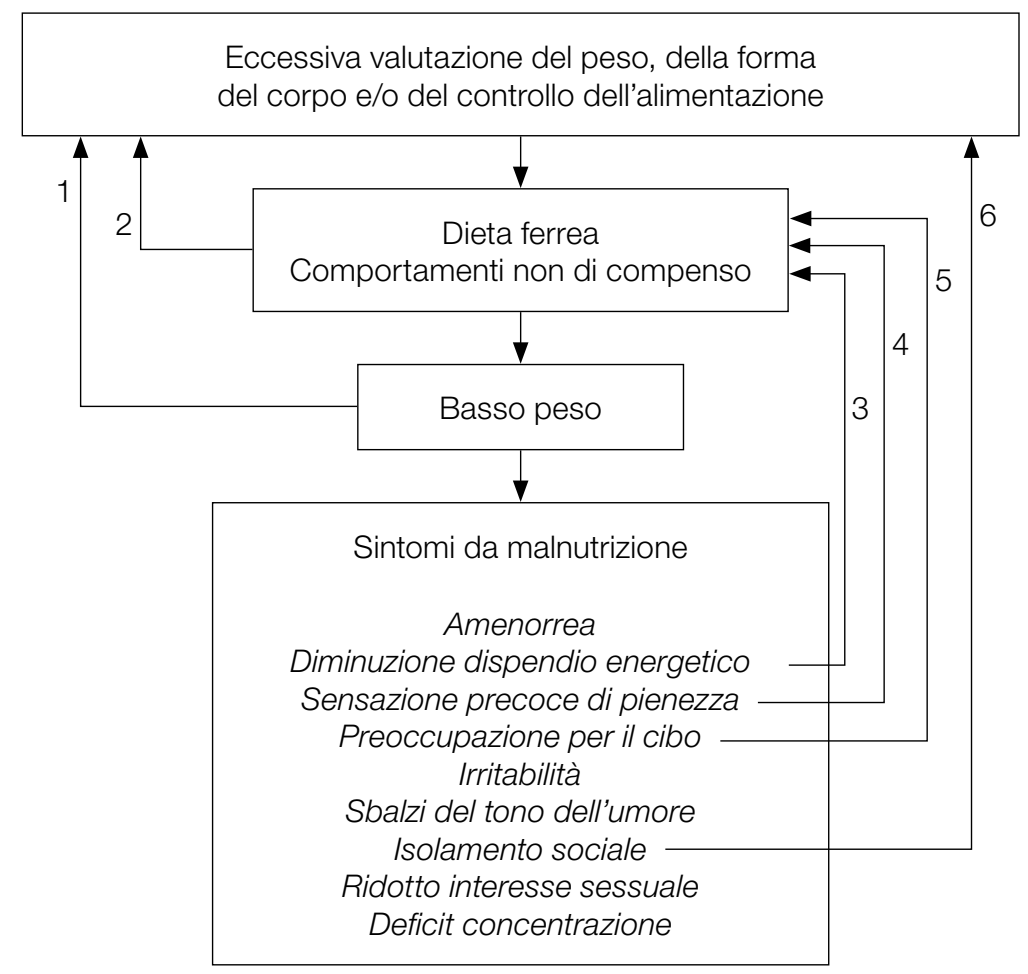

1 = II basso peso non tende ad essere visto come un problema ma come una conquista (in presenza di un'eccessiva valutazione del peso e della forma del corpo).

2 = La dieta ferrea non è vista come un problema perché serve a raggiungere il basso peso ed è considerata la prova della propria forza di volontà.

3 = La diminuzione del dispendio energetico determina un rallentamento nel calo ponderale che fa pensare ad un'incapacità nel controllo del peso e porta a intensificare la restrizione dell'alimentazione.

$4=$ La sensazione di pienezza fa percepire di aver mangiato troppo e porta a restringere ulteriormente l'alimentazione.

$5=$ La preoccupazione per il cibo rende la dieta ancora più rigida.

6 = L'isolamento sociale impedisce di fare delle esperienze che possono aiutare a diminuire l'importanza che si attribuisce al peso, alla forma del corpo e al controllo dell'alimentazione.

Figura 2. Principali meccanismi attraverso cui i sintomi da malnutrizione mantengono la psicopatologia del disturbo dell'alimentazione 
Sebbene la valutazione della presenza dei sintomi da malnutrizione sia molto utile nella pratica clinica, finora i clinici non avevano a disposizione strumenti per valutarli. Per far fronte a questa mancanza abbiamo ideato e validato un nuovo strumento chiamato "Starvation Symptom Inventory (SSI)" che misura i principali sintomi psicosociali e fisici da malnutrizione nei disturbi dell'alimentazione. La descrizione e validazione dello SSI è stata pubblicata in una rivista in lingua inglese (Calugi, Miniati, et al., 2017), ma non è ancora stata resa disponibile in lingua italiana. Scopo di questo articolo è descrivere in lingua italiana il processo di costruzione e validazione dello SSI e di mettere a disposizione dei ricercatori e clinici italiani la versione italiana dello SSI.

\section{Metodi}

\section{Partecipanti}

Campione con anoressia nervosa. Il campione è costituito di 150 pazienti di sesso femminile che hanno soddisfatto i criteri diagnostici del Manuale Diagnostico Statistico dei Disturbi Mentali (DSM-5) (American Psychiatric Association, 2013) per l'anoressia nervosa. La diagnosi DSM-5 è stata eseguita da esperti del campo usando la versione italiana dell'intervista Eating Disorder Examination (EDE) (Calugi, Milanese, et al., 2017). Centoventotto di questi pazienti sono stati reclutati dal reparto di riabilitazione intensiva ospedaliera dei disturbi dell'alimentazione della Casa di Cura Villa Garda e 22 dal centro clinico ambulatoriale ADA di Verona.

Campioni con disturbi dell'alimentazione non sottopeso e disturbo bipolare in fase depressiva. Questi due campioni sono stati inclusi per consentire di analizzare le loro differenze rispetto ai pazienti con anoressia nervosa. Il campione non sottopeso con disturbi dell'alimentazione è costituito da 30 pazienti (13 ospedalieri e 17 ambulatoriali) diagnosticati con l'intervista EDE ( $\mathrm{n}=14$ con bulimia nervosa; $\mathrm{n}=4$ con disturbo da binge-eating ed eccessiva valutazione del peso e della forma del corpo; $\mathrm{n}=12$ con altri disturbi della nutrizione e dell'alimentazione con specificazione). I pazienti con anoressia nervosa atipica sono stati esclusi dallo studio. Il campione con disturbo bipolare in fase depressiva è costituito da 15 pazienti diagnosticati da uno psichiatra senior usando i criteri diagnostici del DSM5 (American Psychiatric Association, 2013). Sono stati esclusi i pazienti con disturbo bipolare in fase depressiva che: (i) erano sottopeso (Indice di Massa Corporea - IMC - $<18.5 \mathrm{~kg} / \mathrm{m}^{2}$ ); (ii) soddisfacevano i criteri diagnostici di un disturbo della nutrizione e dell'alimentazione, incluso il disturbo da binge-eating; (iii) avevano perso almeno il $10 \%$ di peso nei sei mesi precedenti.

Campione di controllo sano. Il campione è costituito da 341 soggetti di sesso femminile (217 reclutati in vari setting della comunità tramite un sondaggio online e 124 tra gli studenti universitari e i membri dello staff). Tutti i partecipanti sani hanno riportato il loro peso e la loro altezza. I soggetti sono stati esclusi se hanno riportato un IMC $<18,5 \mathrm{~kg} / \mathrm{m}^{2}(\mathrm{n}=20)$ e/o un punteggio uguale o superiore a 20 alla versione italiana dell'Eating Attitudes Test-26 (EAT-26) $(\mathrm{n}=15)$ e/o avevano una sospetta diagnosi di disturbo dell'alimentazione perché avevano risposto affermativamente a una o entrambe delle seguenti due domande: "Ha un disturbo dell'alimentazione?" e/o "È in trattamento per un disturbo dell'alimentazione?” $(\mathrm{n}=1)$. I partecipanti hanno dato il consenso informato scritto per l'uso anonimo dei loro dati per scopi clinici e di ricerca. Per quelli di età inferiore ai 18 anni, è stato richiesto un ulteriore consenso informato dai loro genitori.

\section{Misure}

Starvation Symptom Inventory (SSI). Gli item iniziali dello SSI sono stati generati prendendo in considerazione i sintomi da malnutrizione descritti dal Minnesota Starvation Study (Keys et al., 1950), le osservazioni cliniche dei resoconti dei pazienti e le discussioni tra ricercatori e clinici specializzati nei disturbi dell'alimentazione. Il gruppo iniziale di domande è stato discusso tra gli autori al fine di raggiungere un consenso su quali item aggiungere o rimuovere al fine di ottenere una descrizione completa dei sintomi da malnutrizione. Questo processo ha portato ad avere un questionario finale composto da 16 item. La struttura dello SSI rispecchia quella dell'Eating Disorder Examination Questionnaire (EDE-Q) (Calugi, Milanese, et al., 2017), e ai partecipanti viene chiesto di fornire una stima del numero di giorni dei precedenti 28 (quattro settimane), in cui hanno manifestato i sintomi da malnutrizione, su una scala Likert a 7 punti che va da "mai" (0) a "sempre" (6).

Psicopatologia del disturbo dell'alimentazione e caratteristiche psichiatriche generale. Le versioni italiane dell'EDE-Q (Calugi, Milanese, et al., 2017) e del Brief Symptom Inventory (BSI) (De Leo, Frisoni, Rozzini, \& Trabucchi, 1993) sono state usate nel campione con anoressia nervosa per valutare la relazione tra i sintomi da malnutrizione con la psicopatologia del disturbo dell'alimentazione e le caratteristiche psichiatriche generali. 


\section{Analisi statistica}

Per esplorare la validità di costrutto è stata eseguita, separatamente nei campioni con anoressia nervosa e controlli sani, l'analisi delle componenti principali (PCA) con rotazione obliqua (promax) - ipotizzando, quindi, che i fattori identificati sarebbero stati correlati l'un l'altro - e con la normalizzazione di Kaiser (Tabachnick \& Fidell, 2013). Il test di Kaiser-Meyer-Olkin (KMO) è stato utilizzato per valutare l'adeguatezza del campionamento, che è stato considerato adeguato se il KMO era superiore a 0,5. Per garantire un adeguato rapporto soggetto-item, il numero di questionari da somministrare è stato determinato in anticipo ( $\mathrm{n} \mathrm{da}$ 80 a 160), come raccomandato dall'analisi delle componenti principali (Gorusch, 1983). Il numero di fattori da estrarre è stato definito sia ispezionando lo Scree Plot sia considerando la loro interpretabilità e consistenza con i criteri che hanno guidato la costruzione dello strumento. Gli item sono inclusi in un fattore solo se raggiungono una saturazione di 0,40 o maggiore. Anche le correlazioni item-totale sono state esaminate per ogni singolo fattore.

Nel campione con anoressia nervosa sono state eseguite le seguenti analisi: (i) l'alfa di Cronbach è stata calcolata come indicatore di consistenza interna; (ii) le correlazioni di Pearson sono state condotte per valutare la validità convergente. Lo SSI è stato somministrato anche una seconda volta (da una a tre settimane dopo la somministrazione iniziale) a un sottogruppo del campione con anoressia nervosa $(\mathrm{n}=34)$ che non aveva ancora iniziato il trattamento, per consentire il calcolo dell'attendibilità test-retest.
Inoltre, la validità del criterio è stata testata confrontando il punteggio globale dello SSI nei pazienti con anoressia nervosa con i controlli sani, i pazienti con disturbo dell'alimentazione non sottopeso e i pazienti con disturbo bipolare in fase depressiva. Per questo è stata utilizzata l'analisi della varianza (ANOVA) con test post-hoc di Bonferroni.

\section{Risultati}

Validità di costrutto. Nel campione con anoressia nervosa, l'analisi delle componenti principali con rotazione obliqua ha rivelato tre fattori, con autovalori (eigenvalues) superiori alla soglia di 1,00, che spiegano complessivamente il $58,3 \%$ della varianza. Il KMO era 0,88 , indicando che l'analisi delle componenti principali era appropriata per 16 item dello SSI. Ispezionando lo Scree Plot (Figura 3), un chiaro cambiamento di curvatura è stato osservato dopo il primo fattore, suggerendo la presenza di un unico fattore pari al $43,5 \%$ della varianza. La soluzione a un fattore è stata selezionata come la migliore in termini di utilità e interpretabilità dei sintomi da malnutrizione. Un item (numero 15) della soluzione a un fattore presentava un la presenza di un unico livello di saturazione inferiore a 0,40 $(0,16)$ ed è stato rimosso dal questionario. La Tabella 1 mostra le caratteristiche per i rimanenti 15 item; la soluzione finale a fattore singolo rappresentava il $46,4 \%$ della varianza e la saturazione dei 15 item variava da 0,420 a 0,817 . Nel campione di controllo sano, è emerso un pattern di fattori,

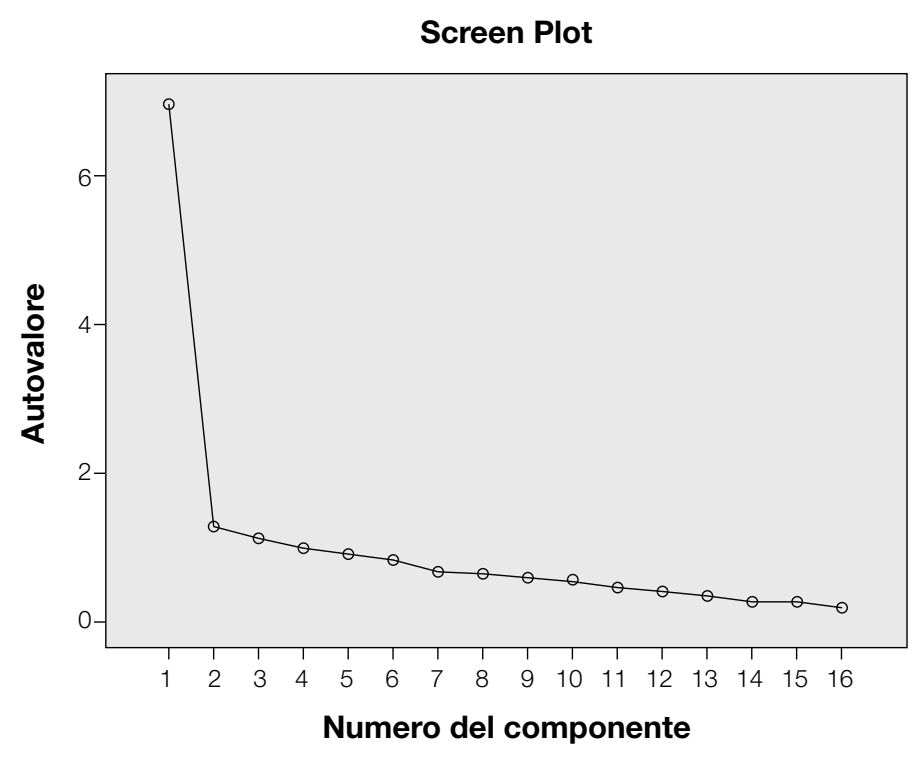

Figura 3. Scree Plot degli autovalori dei 16 item originale dello Starvation Symptom Inventory nel campione con anoressia nervosa 
Tabella 1. I 15 item dello Starvation Symptom Inventory: media (deviazione standard), autovalori, percentuale della varianza, saturazione nel fattore e correlazione item-totale nel campione con anoressia nervosa

\begin{tabular}{|c|c|c|c|c|c|}
\hline & Media (SD)* & Autovalore & $\%$ varianza & Fattore & Item-total \\
\hline 1. Si è preoccupato/a per il cibo? & $5,2(1,7)$ & 6,96 & 46,4 & 1,12 & 0,54 \\
\hline $\begin{array}{l}\text { 2. Ha collezionato ricette, libri di cucina, } \\
\text { menù? }\end{array}$ & $1,6(2,1)$ & 0,605 & 7,5 & 0,420 & 0,37 \\
\hline $\begin{array}{l}\text { 3. Ha incrementato il consumo di caffè, tè o } \\
\text { spezie? }\end{array}$ & $2,7(2,5)$ & 0,96 & 6,4 & 0,555 & 0,51 \\
\hline 4. È stato/a depresso/a? & $3,8(2,0)$ & 0,88 & 5,8 & 0,817 & 0,75 \\
\hline 5. È stato/a ansioso/a? & $4,4(1,9)$ & 0,80 & 5,3 & 0,799 & 0,73 \\
\hline 6. È stato/a irritabile? & $4,1(1,9)$ & 0,79 & 5,1 & 0,755 & 0,69 \\
\hline $\begin{array}{l}\text { 7. È stato/a di umore variabile (eccitato/a e } \\
\text { depresso/a)? }\end{array}$ & $4,2(1,9)$ & 0,61 & 4,0 & 0,763 & 0,70 \\
\hline 8. Si è isolato/a dagli altri? & $3,8(2,1)$ & 0,60 & 4,0 & 0,786 & 0,72 \\
\hline $\begin{array}{l}\text { 9. È diminuita la sua capacità di } \\
\text { concentrazione? }\end{array}$ & $3,6(2,1)$ & 0,52 & 3,4 & 0,706 & 0,65 \\
\hline 10. È stato/a apatico/a? & $3,3(2,2)$ & 0,42 & 2,7 & 0,810 & 0,75 \\
\hline 11. Ha avuto un sonno disturbato? & $3,4(2,2)$ & 0,39 & 2,6 & 0,636 & 0,58 \\
\hline 12. Si è sentito/a debole? & $3,7(2,1)$ & 0,33 & 2,2 & 0,675 & 0,62 \\
\hline $\begin{array}{l}\text { 13. Ha avuto una diminuzione dell'interesse } \\
\text { sessuale? }\end{array}$ & $4,2(2,3)$ & 0,26 & 1,8 & 0,659 & 0,59 \\
\hline 14. Ha avuto freddo? & $4,3(2,0)$ & 0,22 & 1,5 & 0,581 & 0,53 \\
\hline 15. Ha avuto un precoce senso di sazietà? & $3,8(2,3)$ & 0,14 & 0,9 & 0,499 & 0,44 \\
\hline
\end{tabular}

* Misurati su una scala Likert con punteggi da 0 a 6

pari al 60,9\% della varianza, tuttavia il Scree Plot ha suggerito una soluzione a un fattore, pari al $37,2 \%$ della varianza. L'item 2 mostrava una saturazione inferiore a $0,40(\mathrm{r}=$ $0,021)$ nella soluzione a un fattore mentre l'item 15 aveva una saturazione di 0,572. Il restante pattern di fattori era lo stesso nei campioni con anoressia nervosa e nei controlli sani.

Affidabilità. La consistenza interna (alpha di Cronbach) dello SSI era 0,91 e l'attendbilità test-retest era $\mathrm{r}=0,90, \mathrm{p}$ $<0,001$.

Analisi di correlazione. Le analisi di correlazione hanno evidenziato che il punteggio medio dello SSI non era significativamente correlato con l'età $(\mathrm{r}=-0,13, \mathrm{p}=0,114)$ o la durata della malattia $(r=-0,060, p=0,467)$ e solo debolmente con l'IMC ( $r=-0,22, p=0,007)$. Al contrario, il punteggio globale dello SSI era significativamente correlato con il punteggio globale dell'EDE-Q $(r=0,75, p<0,001)$ e delle sottoscale (restrizione $r=0,60, p<0,001$; preoccupazione per l'alimentazione $\mathrm{r}=0,69, \mathrm{p}<0,001$; preoccupazione per il peso $r=0,72, p<0,001$; preoccupazione per la forma del corpo $\mathrm{r}=0,74, \mathrm{p}<0,001)$ e con il punteggio globale del BSI $(r=0,82, p<0,001)$. Questi risultati forniscono evidenza della validità convergente.

Confronti delle medie di gruppo. La Tabella 2 mostra le caratteristiche basali e il punteggio globale dello SSI nei quattro campioni. I pazienti con anoressia nervosa avevano un'età significativamente più bassa di quelli con disturbo bipolare in fase depressiva e del campione di controllo sano, un peso corporeo e un IMC significativamente più basso e un punteggio globale dello SSI superiore degli altri tre gruppi. Inoltre, il punteggio globale dello SSI era significativamente più alto nei pazienti con disturbo dell'alimentazione non sottopeso rispetto a quelli con disturbo bipolare in fase depressiva e ai controlli sani. Infine, i pazienti con disturbo bipolare in fase depressiva avevano un punteggio più alto dello SSI rispetto ai controlli sani. La differenza significativa tra i quattro gruppi è confermata controllando le analisi per l'età e l'IMC. 
Tabella 2. Caratteristiche basali e punteggio globale dello SSI nei pazienti con anoressia nervosa, disturbo dell'alimentazione non sottopeso, disturbo bipolare in fase depressiva e nei controlli sani

\begin{tabular}{l|c|c|c|c|c|c}
\hline & $\begin{array}{c}\text { Anoressia } \\
\text { nervosa } \\
(\mathbf{n = 1 5 0 )}\end{array}$ & $\begin{array}{c}\text { Disturbo } \\
\text { dell'alimentazione } \\
\text { non sottopeso } \\
\mathbf{( n = 3 0 )}\end{array}$ & $\begin{array}{c}\text { Disturbo } \\
\text { bipolare in fase } \\
\text { depressiva } \\
\mathbf{( n = 1 5 )}\end{array}$ & $\begin{array}{c}\text { Controlli } \\
\mathbf{s a n i} \\
\mathbf{( n = 3 4 1 )}\end{array}$ & valore-p & $\begin{array}{c}\text { Confronto } \\
\text { post-hoc }\end{array}$ \\
\hline Età (anni) & $25,2(9,4)$ & $28,3(11,6)$ & $32,2(9,1)$ & $30,0(9,9)$ & $<0,001$ & $a<c, d$ \\
\hline Peso corporeo (kg) & $38,8(5,8)$ & $71,6(26,8)$ & $64,3(11,2)$ & $61,2(9,8)$ & $<0,001$ & $\begin{array}{c}a<b, c, d \\
b>d\end{array}$ \\
\hline $\begin{array}{l}\text { Indice di massa } \\
\text { corporea (kg/m²) }\end{array}$ & $14,7(1,8)$ & $26,6(9,0)$ & $24,1(2,2)$ & $22,0(2,7)$ & $<0,001$ & $\begin{array}{c}a<b, c, d \\
b>d\end{array}$ \\
\hline $\begin{array}{l}\text { Punteggio globale } \\
\text { SSI }\end{array}$ & $55,1(20,4)$ & $46,9(22,9)$ & $26,3(8,4)$ & $10,4(9,7)$ & $<0,001$ & $\begin{array}{c}a>b, c, d \\
b>c, d \\
c>d\end{array}$ \\
\hline
\end{tabular}

$\mathrm{a}=$ pazienti con anoressia nervosa

$\mathrm{b}=$ pazienti con disturbo dell'alimentazione non sottopeso

$\mathrm{c}=$ pazienti con disturbo bipolare in fase depressiva

$\mathrm{d}=$ controlli sani

\section{Discussione}

Lo studio si è posto l'obiettivo di descrivere la progettazione e la validazione della versione italiana dello SSI in un gruppo di pazienti con anoressia nervosa confrontando i loro punteggi con controlli sani, con pazienti con disturbo dell'alimentazione non sottopeso e con disturbo bipolare in fase depressiva. Dai risultati dallo studio si possono trarre tre conclusioni principali.

La prima conclusione riguarda la validità di costrutto. Nello specifico, l'analisi delle componenti principali ha indicato che la soluzione a un fattore è la migliore nei pazienti con anoressia nervosa, rappresentando oltre il $46 \%$ della varianza. Risultati simili sono stati ottenuti nei controlli sani. Inoltre, l'analisi ha consentito l'identificazione di un item ("Ha avuto un aumento della fame") con una saturazione molto bassa che è stato omesso dal questionario finale che, perciò, è costituito da 15 item (vedi Appendice 1).

La seconda conclusione è che la versione finale dello SSI ha mostrato una buona coerenza interna e attendibilità test-retest. Ciò indica che gli item misurano lo stesso costrutto generale e che lo strumento è stabile nel tempo.

La terza conclusione riguarda la validità convergente e divergente. In particolare, il punteggio globale dello SSI è risultato significativamente associato con la psicopatologia del disturbo dell'alimentazione e la psicopatologia generale e ha mostrato punteggi significativamente più alti nei pazienti con anoressia nervosa, rispetto ai controlli sani, ai pazienti con disturbo dell'alimentazione non sottopeso e con disturbo bipolare in fase depressiva. Ciò indica che i sintomi da malnutrizione valutati sono correlati con la psicopatologia, ma sono specifici per i pazienti sottopeso. Inoltre, la piccola ma significativa differenza tra i pazienti con anoressia nervosa e i disturbi dell'alimentazione non sottopeso potrebbe indicare che alcuni sintomi inclusi nello SSI non sono specifici della malnutrizione, ma sono invece caratteristiche della restrizione dietetica cognitiva (Dalle Grave et al., 2007).

I punti di forza dello studio sono l'uso di un ampio numero di pazienti con anoressia nervosa, l'inclusione di un ampio numero di controlli sani, e due campioni di pazienti con disturbo dell'alimentazione e di pazienti con disturbo bipolare in fase depressiva, che ha consentito l'esame della validità psicometrica e clinica dello strumento e ci permette di escludere la possibilità che alcuni sintomi siano legati alla psicopatologia del disturbo dell'alimentazione o alla sintomatologia depressiva piuttosto che alla malnutrizione.

I limiti dello studio riguardano l'incapacità di valutare la validità concorrente con altri strumenti esistenti, poiché non sono disponibili questionari validati per indagare i sintomi da malnutrizione. Inoltre, la dimensione del campione clinico relativamente limitata ci ha impedito di utilizzare l'analisi fattoriale confermativa o l'Item Response Theory per esaminare le prestazioni degli item in 
maggiore dettaglio. Infine, il basso numero di pazienti con disturbo dell'alimentazione non sottopeso e con disturbo bipolare in fase depressiva potrebbe limitare la rappresentatività di questi campioni nelle rispettive popolazioni.

In conclusione, lo SSI sembra essere uno strumento promettente per misurare i sintomi da malnutrizione nei pazienti con anoressia nervosa. Può essere facilmente integrato nella pratica clinica di routine per valutare i sintomi da malnutrizione nei pazienti sottopeso con disturbi dell'alimentazione e per valutare i loro cambiamenti durante il processo di recupero del peso.

\section{Referenze}

American Psychiatric Association. (2013). Diagnostic and statistical manual of mental disorders, (DSM-5). Arlington: American Psychiatric Publishing.

Calugi, S., Milanese, C., Sartirana, M., El Ghoch, M., Sartori, F., Geccherle, E., ... Dalle Grave, R. (2017). The Eating Disorder Examination Questionnaire: reliability and validity of the Italian version. Eating and Weight Disorders, 22(3), 509-514. doi:10.1007/ s40519-016-0276-6

Calugi, S., Miniati, M., Milanese, C., Sartirana, M., El Ghoch, M., \& Dalle Grave, R. (2017). The Starvation Symptom Inventory: Development and psychometric properties. Nutrients, 9(9), 967. doi:10.3390/nu9090967

Dalle Grave, R., Calugi, S., \& Sartirana, M. (2018). Manuale di terapia cognitivo comportamentale dei disturbi dell'alimentazione nell'adolescenza (CBT-Ea). Dal trattamento ambulatoriale al ricovero riabilitativo. Verona: Positive Press.

Dalle Grave, R., Di Pauli, D., Sartirana, M., Calugi, S., \& Shafran, R. (2007). The interpretation of symptoms of starvation/severe dietary restraint in eating disorder patients. Eating and Weight Disorders, 12(3), 108-113.
Dalle Grave, R., Pasqualoni, E., \& Marchesini, G. (2011). Symptoms of starvation in eating disorder patients. In V. R. Preedy (Ed.), Handbook of Behavior, Food and Nutrition (pp. 2259-2269). New York: Springer Science+Business Media.

De Leo, D., Frisoni, G. B., Rozzini, R., \& Trabucchi, M. (1993). Italian community norms for the Brief Symptom Inventory in the elderly. British Journal of Clinical Psychology, 32(2), 209-213. doi:10.1111/j.2044-8260.1993.tb01045.x

Fairburn, C. G. (2008). Cognitive behavior therapy and eating disorders. New York: Guilford Press.

Garner, D. M. (1977). Psychoeducational principles in treatment. In D. M. Garner \& P. Garfinkel (Eds.), Handbook of treatment for eating disorders (pp. 145177). New York: Guilford Press.

Gorusch, R. L. (1983). Factor Analysis. (2nd ed). Hillsdale, NJ: Lawrence Erlbaum Associates.

Kalm, L. M., \& Semba, R. D. (2005). They starved so that others be better fed: remembering Ancel Keys and the Minnesota experiment. Journal of Nutrition, 135(6), 1347-1352. doi:10.1093/jn/135.6.1347

Keys, A., Brozek, J., Henschel, A., Mickelsen, O., \& Taylor, H. (1950). The Biology of Human Starvation. Minneapolis: University of Minnesota Press.

Shafran, R., Fairburn, C. G., Nelson, L., \& Robinson, P. H. (2003). The interpretation of symptoms of severe dietary restraint. Behaviour Research and Therapy, 41(8), 887-894.

Tabachnick, B. G., \& Fidell, S. L. (2013). Principal components and factor analysis. Using multivariate statistics. Boston: Pearson. 


\section{Appendice 1}

\section{Starvation Symptom Inventory (SSI)}

Istruzioni. Le seguenti domande riguardano le ultime quattro settimane (28 giorni). È pregato di leggere attentamente ogni domanda. La preghiamo di rispondere a tutte le domande. Grazie

\begin{tabular}{|c|c|c|c|c|c|c|c|}
\hline Quante volte negli ultimi 28 giorni & Mai & $\begin{array}{l}1-5 \\
\text { giorni }\end{array}$ & $\begin{array}{l}6-12 \\
\text { giorni }\end{array}$ & $\begin{array}{l}13-15 \\
\text { giorni }\end{array}$ & $\begin{array}{l}16-22 \\
\text { giorni }\end{array}$ & $\begin{array}{l}23-27 \\
\text { giorni }\end{array}$ & $\begin{array}{l}\text { Ogni } \\
\text { giorno }\end{array}$ \\
\hline \multicolumn{8}{|l|}{ Si è preoccupato/a per il cibo? } \\
\hline \multicolumn{8}{|c|}{ Ha collezionato ricette, libri di cucina, menu? } \\
\hline \multicolumn{8}{|c|}{ Ha incrementato il consumo di caffè, tè o spezie? } \\
\hline \multicolumn{8}{|l|}{ È stato/a depresso/a? } \\
\hline \multicolumn{8}{|l|}{ È stato/a ansioso/a? } \\
\hline \multicolumn{8}{|l|}{ È stato/a irritabile? } \\
\hline \multicolumn{8}{|c|}{ È stato/a di umore variabile (eccitato/a e depresso/a)? } \\
\hline \multicolumn{8}{|l|}{ Si è isolato/a dagli altri? } \\
\hline \multicolumn{8}{|c|}{ È diminuita la sua capacità di concentrazione? } \\
\hline \multicolumn{8}{|l|}{ È stato/a apatico/a? } \\
\hline \multicolumn{8}{|l|}{ Ha avuto un sonno disturbato? } \\
\hline \multicolumn{8}{|l|}{ Si è sentito/a debole? } \\
\hline \multicolumn{8}{|c|}{ Ha avuto una diminuzione dell'interesse sessuale? } \\
\hline \multicolumn{8}{|l|}{ Ha avuto freddo? } \\
\hline \multicolumn{8}{|l|}{ Ha avuto un aumento della fame? } \\
\hline Ha avuto un precoce senso di sazietà? & & & & & & & \\
\hline
\end{tabular}

